\title{
Factors associated with sweet taste sensitivity in patients with type 2 diabetes mellitus (T2DM)
}

\author{
Vidanage $D^{1}$, Prathapan $s^{2}$, Wasalathanthri $s^{3}$, Hettiarachchi $P^{4}$
}

\begin{abstract}
Introduction: Taste sensitivity plays a vital role in determining food preferences. Evidence suggests that patients with diabetes are less sensitive to sweet taste. Increased sugar consumption due to low sensitivity adversely affect the glycemic control in Type 2Diabetes Mellitus (T2DM). Although the factors associated with taste sensitivity have been assessed in healthy individuals, it is less reported in T2DM. Thus, the aim of this study was to determine the factors associated with taste sensitivity for sucrose in patients with T2DM.
\end{abstract}

Method: A sample of 111 patients, aged between 35-60 years, having T2DM for > 5 years were considered. Baseline sociodemographic and anthropometric parameters were obtained. Supra-threshold intensity ratings for a range of sucrose concentrations were assessed using 'general Labeled Magnitude Scale'. HbA1c was determined at recruitment. Data was analyzed using Multiple Linear Regression.

Results: Results showed that in patients with T2DM,a significant association was evident between the suprathreshold intensity ratings for the highest concentration of sucrose $(2.02 \mathrm{M})$ tested and the waist circumference $(p=0.022)$, hip circumference $(p=0.039)$ and waist-hip ratio $(p=0.022)$. None of the other factors were significantly associated with either the highest or any other concentration tested.

Conclusion: Reduction in waist circumference, hip circumference and waist hip ratio is likely to improve the sweet taste sensitivity in T2DM patients and vice versa. Maintaining these parameters within healthy limits may helpachieving a better glycemic profile through reducing the sugar consumption in patients with T2DM.

\section{Introduction}

Type 2 diabetes mellitus (T2DM) has become a major public health concern in South Asia.1More than a decade ago, it was proven that one in five adults in Sri Lanka had either diabetes or pre-diabetes ${ }^{2}$ with no evidence of improvement regarding the situation currently.A high prevalence of diabetes

1. Department of Nursing \& Midwifery, Faculty of Allied Health Sciences, General Sir John Kotelawala Defence University

2. Department of Community Medicine, Faculty of Medical Sciences, University of Sri Jayewardenepura

3. Department of Physiology, Faculty of Medicine, University of Colombo

4. Department of Physiology, Faculty of Medical Sciences, University of Sri Jayewardenepura

Corresponding Author:

Dinithi Vidanage

E mail: dinithividanage@gmail.com

(iD https://orcid.org/0000-0002-0837-7270 reported in urban South Asia has been associated with sedentary lifestyle,excessive consumption of energy dense food including sugar sweetened beverages andfood rich in sugar and saturated fats. ${ }^{3}$ Consumption of sugar exceeding the healthy limits disrupts the glycemic control in patients with diabetes, causing an impairment of sweet taste sensitivity. ${ }^{4}$ Most sugar sweetened beverages and sweet food contain a high concentration of sucrose, and determining the supra-threshold intensity ratings for sucrose will reflect a person'sactual ability toperceive different concentrations of sweet taste. The ratings given by patients with T2DM for high concentrations of sucrose were found to be lower compared to those given by normoglycemics. ${ }^{5}$ Thu $\mathrm{s}$, this may imply the reduced sweet taste sensitivity of patients with diabetes,driving them to consume excessive amounts of sugarwhich in turn making them overweight or obese.There is inconsistent evidence regarding body mass index (BMI) and sweet taste sensitivity in healthy adults with some reports showing an inverse relationship6while others show no association ${ }^{7}$.In addition, several investigators haveexplored the association between sweet taste sensitivityand other factors such as age, gender, 
glycemic control, etc $\cdot 8,9,11,12$ with findings been inconsistent amongst the studies. 8,9

Identifying the taste sensitivity for sucrose in patients with diabetes may provide useful information when recommending dietary adjustments. New knowledge about the predictors of sweet taste sensitivity will be useful to suggest strategies to overcome excessive sugarconsumption in patients with diabetes in their day to day life. Thus, the present study was aimed at investigating the factors associated with taste sensitivity for sucrose in patients with T2DM.

\section{Methodology}

\section{Study design and setting}

This study is a cross-sectional component of a randomized controlled trial conducted at the Department of Physiology, University of Sri Jayewardenepura to assess the impact of home-based exercises on taste perception in patients with T2DM. Baseline data of this clinical trial have been considered in the present article.

\section{Study participants}

Patients who attended the diabetic clinic at the Family Practice Center, University of Sri Jayewardenepura were recruited by systematic random sampling method. Baseline data of a total of 111 patients, aged between $35-60$ years, with a history of T2DM for more than 5 years, having $\mathrm{HbA} 1 \mathrm{c}$ between 6.6\%-9.9\% were recruited. Patients who were smokers, who had oral ulcers and who chew betel were excluded as these affect the taste perception. In addition, those who were on medications that make significant changes in the taste perception were also excluded.

\section{Data collection}

After obtaining the written informed consent, data on socio-demographic and clinical characteristics such as age, gender, marital status, educational level and duration of diabetes was obtained by an interviewer administered questionnaire. A pilot test was conducted on 10 participants who had similar inclusionexclusion criteria but were not a part of the study. The study was approved by the Ethics Review Committee of Faculty of Medical Sci-

ences, University of Sri Jayewardenepura (No. 10/18) and registered in the Clinical Trials Registry, Sri Lanka (SLCTR/2015/029).

Supra-threshold intensity ratings for sucrose were obtained on subjects who were asked to abstain from food, smoking, alcohol and betel chewing from $10 \mathrm{pm}$, the previous day. A standard breakfast comprising of a plantain and 2 slices of brown bread with margarine was given 1 hour before the sensitivity testing to standardize their level of satiety. Suprathreshold intensity ratings were assessed by using a series of sucrose solutions (i.e. $2.02 \mathrm{M}, 0.64 \mathrm{M}, 0.2 \mathrm{M}$, $0.064 \mathrm{M}, 0.0202 \mathrm{M}, 0.0064 \mathrm{M})$.The patients were instructed to rate the intensity of sweet taste on a general Labeled Magnitude Scale (gLMS)which is a $100 \mathrm{mmScale}$ ranging from no sensation $(0 \mathrm{~mm})$ to the strongest imaginablesensation $(100 \mathrm{~mm})$.The mean supra-threshold intensity rating was calculated for each concentration after 3 consecutive tests.4To train the use of gLMS and to control idiosyncratic scale usage, participants were trained by getting them to rate the heaviness of six visually identical weights (i.e.sand and stone filled opaque bottles of weights 225, 380, 558, 713, 870, and 999 g, completely wrapped with foil papers).7Anthropometric parameters i.e. weight, height, waist circumference (WC) and hip circumference (HC)were obtained before taste testing. BMI, waist-hip ratio (WHR) and waist height ratio (WHtR) were calculated accord-

Table 1. Characteristics of study participants

\begin{tabular}{ll} 
Variable & $\begin{array}{l}\text { Participants } \\
(\mathrm{n}=111)\end{array}$ \\
\hline Age (years)* & $49.75 / \pm 6.1$ \\
\hline $\begin{array}{l}\text { Gender } \\
\text { Fale }\end{array}$ & $57(51.3 \%)$ \\
& $54(48.7 \%)$ \\
\hline Education level & \\
No education & 0 \\
Grade 1-5 & 0 \\
Grade 6-10 & $29(26.1 \%)$ \\
Up to Ordinary level & $25(22.5 \%)$ \\
Up to Advanced Level & $49(44.2 \%)$ \\
Graduate & $08(7.2 \%)$ \\
\hline Marital status & \\
Married & $101(91 \%)$ \\
Unmarried & $10(9 \%)$ \\
\hline Duration of T2DM (years)* & $8.04 / \pm 4.4$ \\
BMI (kg/m2) & $24.67 / \pm 3.5$ \\
\hline Waist Circumference (cm)* & $85.92 / \pm 10.8$ \\
\hline Hip Circumference (cm)* & $86.56 / \pm 12.1$ \\
\hline Waist-Hip Ratio & 0.9 \\
\hline Waist-Height Ratio & 0.5 \\
\hline HbA1c (\%) & $7.85 / \pm 1.5$ \\
\hline *Mean / \pm Standard deviation & \\
\hline
\end{tabular}

ingly. Glycated hemoglobin (HbA1c) was tested on all participants to assess their glycemic control.

\section{Statistical analysis}

Descriptive statistics were presented as numbers and percentages,and means and standard deviations (SDs). The factors associatedwithsweet taste sensitivity for sucrose weredetermined by Multiple Linear Regression in SPSS version 23.0. The determinants for Multiple Linear Regression model predicting taste sensitivity for sucrose includedgender, age, duration of diabetes, HbA1c level, BMI, WC, HC,WHR andWHtR. Results

Socio demographic, anthropometric and clinical characteristics of the study participants are shown in Table 1. A little more than half of the cohort were 
males (51.3\%). Mean age of the participants was 49.7 years (SD +/-6.1) and the mean duration of T2DM was about 8 years. In most participants, the anthropometric parameters (BMI, WC, HC, WHR) have exceeded the normal cutoffs as per the Asian standards.10MostT2DM patients showed evidence of a poor glycemic control.

The sweet taste was highest in the $2.02 \mathrm{M}$ sucrose solution and decreased gradually along the series of $0.64 \mathrm{M}, 0.2 \mathrm{M}, 0.064 \mathrm{M}, 0.0202 \mathrm{M}$ concentrations with0.0064M having the lowest sweet taste. Mean (+/-SD) supra-threshold intensity ratings given for sucrose solution. A significant regression equation was found for $2.02 \mathrm{M}$ sucrose solution [ $\mathrm{F}(11,99)$ $=2.116, \mathrm{p}(0.026)]$ which is the highest sucroseconcentration tested, with an R2 of 0.19 .

Participants predicted that sensitivity for the highest sweet taste is equal to 435-429.5 (WHR)4.41(HC)-4.86(WC) where HC and WC are measured in centimeters. Participants' taste sensitivity for $2.02 \mathrm{M}$ solution was increased by 4.41 and 4.86 units for each $1 \mathrm{~cm}$ decrease of $\mathrm{HC}$ and $\mathrm{WC}$ respectively. Moreover, it was observed that anthropometric parameters were negatively correlated with taste sensitivity for almost all sucrose solutions in

Table 2. Supra-threshold intensity ratings given on the gLMS for different concentrations of sucrose $(n=111)$

\begin{tabular}{|lcccccc|}
\hline \multicolumn{7}{|c|}{ Sucrose concentrations } \\
\hline & $2.02 \mathrm{M}$ & $0.64 \mathrm{M}$ & $0.2 \mathrm{M}$ & $0.064 \mathrm{M}$ & $0.0202 \mathrm{M}$ & $0.0064 \mathrm{M}$ \\
\hline $\begin{array}{l}\text { Mean supra-threshold } \\
\text { intensity ratings(mm) }\end{array}$ & 75.57 & 61.18 & 36.95 & 12.31 & 4.92 & 4.15 \\
\hline SD (+/-) & 19.46 & 22.59 & 20.07 & 11.54 & 6.28 & 6.43 \\
\hline
\end{tabular}

Table 3. Factors associated with sweet taste sensitivity in participants( $n=111)$

\begin{tabular}{|c|c|c|c|c|c|c|}
\hline \multirow[t]{2}{*}{ Model } & \multirow[t]{2}{*}{$\begin{array}{l}\text { Unstandardized } \\
\text { coefficient }\end{array}$} & \multicolumn{2}{|c|}{$\begin{array}{l}\text { Standardized } \\
\text { coefficient }\end{array}$} & \multicolumn{3}{|c|}{$\begin{array}{l}\text { 95\% Confidence interval for } \\
\text { B }\end{array}$} \\
\hline & & $\beta$ & $\mathrm{t}$ & $\underset{\text { value }}{\mathrm{p}}$ & $\begin{array}{l}\text { Lower } \\
\text { bound }\end{array}$ & $\begin{array}{l}\text { Upper } \\
\text { bound }\end{array}$ \\
\hline (Constant) & 435.066 & & 0.486 & 0.021 & 67.62 & 802.5 \\
\hline Gender & 5.972 & 0.033 & & 0.132 & -1.83 & 13.78 \\
\hline Age & 0.487 & 0.057 & & 0.130 & -1.14 & 1.12 \\
\hline Duration of T2DM & 0.427 & 0.200 & & 0.315 & -0.41 & 1.26 \\
\hline $\mathrm{HbA1c}$ & -1.314 & -0.077 & & 0.274 & -1.05 & 3.68 \\
\hline $\mathrm{BMI}$ & -0.227 & -0.013 & & 0.725 & -1.50 & 1.05 \\
\hline WC & -4.865 & -0.665 & & $0.022^{*}$ & 0.704 & 9.02 \\
\hline $\mathrm{HC}$ & -4.418 & -0.478 & & $0.039 *$ & -8.608 & -0.22 \\
\hline WHR & -429.557 & -0.299 & & $0.022^{*}$ & 796.7 & -62.31 \\
\hline WHtR & -38.811 & -0.154 & & 0.436 & -137.5 & 59.73 \\
\hline $\mathrm{R}$ & 0.436 & & & & & \\
\hline R square (R2) & 0.19 & & & & & \\
\hline Adjusted $R$ square & 0.1 & & & & & \\
\hline F ratio & 2.116 & & & & & \\
\hline $\begin{array}{l}\text { p value } \\
\text { (Regression model) }\end{array}$ & $0.026^{*}$ & & & & & \\
\hline
\end{tabular}

different concentrations are shown in Table 2.

Dependent variable: Supra-threshold intensity ratings for 2.02M sucrose solution

Independent variables: Gender, Age, Duration of T2DM, HbA1c, body mass index (BMI), waist circumference (WC), hip circumference $(\mathrm{HC})$, waist hip ratio (WHR) and waist-height ratio (WHtR). Level of significance $p<0.05^{*}$

Table 3 shows the results of the regression analysis of the factors predicting taste sensitivity for $2.02 \mathrm{M}$ the tested range. None of the other factors showed significant regression relationships for taste sensitivity for sucrose in the participants.

\section{Discussion}

In the present study, the factors associated with taste sensitivity for sucrose were assessed with six different concentrations of sucrose solutions ranging from the highest to the lowest in patients with T2DM. The factors assessed were gender, age, duration of diabetes, $\mathrm{HbA} 1 \mathrm{c}$ and anthropometry. To our understanding, this is the first comprehen- 
sive study investigating multiple factors associated with taste sensation in Sri Lankan patients with T2DM. Overall, the current findings indicated that the supra-threshold intensity ratings for the highest sucrose concentration was significantly associated with WC, HC and WHRwhile no associations observed with any other factor assessed. Observing an association for the highest sucrose concentration is a crucial finding, since most sweet foods such as cakes, biscuits and sugar sweetened beveragescontain a high concentration of sucrose. Determining the factors that predict taste sensitivity is important when considering sweet food consumption.

Factors such as gender and age have been studied extensively in relation to sweet taste sensitivity. $\mathrm{Yu}$ et al. ${ }^{11}$ showed that diabetic males are less sensitive to sucrose compared to the females. However, we did not observe a significant association between gender and sweet taste sensitivity in our group. The reason for this difference may be attributed to the difference in the psychophysical measure used in determining the taste sensitivity. Yu and co-authors ${ }^{11}$ have used the detection threshold for sucrose,while we used the supra-threshold intensity ratingswhich gives a better estimation of the taste sensitivity. Another study by Mojetet al. ${ }^{12}$ revealed that older men (60-75years) were less sensitive to sucrose compared to youngercounterparts (19-33years). A review by Sergiet al.9stated that, aging may coincide with a declining gustatory function that can affect dietary intake and elderly may prefer strong flavors leading to negative health consequences. In the present study, the participants belonged to a narrow age range of $35-60$ years which wasprobably not sufficient to demonstrate a significant association with taste sensitivity. The most likely reasons for these contradictory findings may be due to the differences in the technique of assessing psychophysics of taste and participant characteristics. In fact, most of these studies have been conducted with healthy individuals belonging to a wide age range and mostly used the detection/recognition thresholds to determine the taste sensitivity. Further, in our study, we have focused on multiple factors associated with sweet taste sensitivity rather than confining to a single factor. However, considering the previous findings, it is justifiable to consider gender and age as contributory factors that can alter sweet taste sensitivity in patients with diabetesspecially when the dietary recommendations are planned. In addition, our cohort did not show a significant association between taste sensitivity and other factors such asduration of diabetes and glycemic control. Similar to our findings, $\mathrm{Yu}$ and co- authors11also reported that duration of diabetes and glycemic profile (Fasting blood glucose \& HbA1c)were not associated with sweet taste sensitivity in T2DM.
The association between anthropometry and taste sensitivity has long been an area of interest, but with mixed scientific evidence. Observations on the relationship between sweet taste thresholds and obesity are contradictory. Donaldson et al. ${ }^{13}$ reported no significant difference in sweet taste thresholdsin obese femalescompared to normal weight equivalents. It is observed that most of these studies have assessed the sweet taste sensitivity only in relation to BMI. However, unlike other studies, the present study highlights the association between sweet taste sensitivity assessed by supra-threshold intensity ratings for sucrose and multiple anthropometric parameters which includes WC, HC, WHR andWHtR. Umabikiet al. ${ }^{14}$ reported that there is an association between sweet taste sensitivity and serum leptin levels assessed in a group of obese females. The results showed a significant association between reduced levels of serum leptin and increased sensitivity to sweet taste. In addition, Yu et al. ${ }^{11}$ showed that BMI and plasma triglyceride levels are positively correlated with detection threshold for sucrose in diabetics indicating less sensitivity. Thus, the mechanism for low sensitivity to sweet taste in obese may be attributed to high serum leptin levels. Even though we have not assessed the hormone levels, the findings in the present study have a major clinical implication suggesting that diabetics who are overweight/obese are likely to be less sensitive to sweet taste which may lead to over consumption of sweet taste foods. ${ }^{15}$ This may be a vicious cycle since increase inWC, $\mathrm{HC}$ and WHR may result in decreasing the sweet taste sensitivity which in turn increase the sweet food consumption which will lead to further alterations in the above anthropometric parameters in patients with diabetes.

\section{Conclusion}

To our knowledge, this is the first study to assess multiple factors associated with sweet taste sensitivity for sucrose in patients with T2DMin Sri Lanka. This investigation confirms that anthropometric parameters i.e. WC, HC, WHR are significantly associated with taste sensitivity for sucrose in T2DM patients. The findingsimply that reduction of these parameters is likely to improve the sweet taste sensitivity in patients with diabetes and vice versa. Planning life style interventions to maintain anthropometric parameters within healthy limits may lead to better glycemic profile through increased taste sensitivity and good dietary practices.

\section{Conflicts of interest}

Authors declare that they have no conflicts of interest

\section{Acknowledgement}

We gratefully acknowledge the financial support given by University of Sri Jayewardenepura for this 
study (Research grant - ASP/06/RE/MED/2014/15 and ASP/01/RE/MED/2017/31).

\section{References}

1. Hills AP, Arena R, Khunti K, Yajnik CS, Jayawardena $\mathrm{R}$, Henry $\mathrm{CJ}$, et al. Epidemiology and determinants of type 2 diabetes in south Asia. Lancet Diabetes Endocrinol. 2018 Dec;6(12):966-78.

2.KatulandaP, ConstantineGR, Mahesh JG, Sheriff R, Seneviratne, RDA, Wijeratne S, Wijesuriya M, McCarthyMI, Adler Al, and MatthewsDR, Prevalence and projections of diabetes and pre-diabetes in adults in Sri Lanka-Sri Lanka Diabetes, Cardiovascular Study (SLDCS). Diabetic Medicine, 25: 10621069. doi:10.1111/j.1464-5491.2008.02523.

3. Feng L, Naheed A, de Silva HA, Jehan I, Raqib R, Islam MT, et al. Regional Variation in Comorbid Prediabetes and Diabetes and Associated Factors among Hypertensive Individuals in Rural Bangladesh, Pakistan, and Sri Lanka. J Obes. 2019 Apr 30;2019:1-11.

4. Green BG, Lim J, Osterhoff F, Blacher K, Nachtigal D. Taste mixture interactions: Suppression, additivity, and the predominance of sweetness. Physiol Behav. 2010 Dec 2;101(5):731-7.

5. Wasalathanthri S, Hettiarachchi P, Prathapan S. Sweet taste sensitivity in pre-diabetics, diabetics and normoglycemic controls: a comparative cross sectional study. BMC Endocr Disord. 2014 Dec;14(1):67.

6. Pasquet $P$, Laure Frelut $M$, Simmen $B$, Marcel Hladik C, Monneuse M-O. Taste perception in massively obese and in non-obese adolescents. Int J Pediatr Obes. 2007 Jan;2(4):242-8.

7. Low JY, Lacy KE, McBride RL, Keast RS. Carbohydrate Taste Sensitivity Is Associated with Starch Intake and Waist Circumference in Adults. J Nutr. 2017 Dec;147(12):2235-42.

8. Yoshinaka M, Ikebe K, Uota M, Ogawa T, Okada T, Inomata $\mathrm{C}$, et al. Age and sex differences in the taste sensitivity of young adult, young-old and old-old Japanese: Age and sex differences in taste sense. Geriatr Gerontol Int. 2016 Dec;16(12):1281-8.

9. Sergi G, Bano G, Pizzato S, Veronese N, Manzato E. Taste loss in the elderly: Possible implications for dietary habits. Crit Rev Food Sci Nutr. 2017 Nov 22;57(17):3684-9.

10. Lim JU, Lee JH, Kim JS, Hwang YI, Kim T-H, Lim
SY, et al. Comparison of World Health Organization and Asia-Pacific body mass index classifications in COPD patients. Int J Chron Obstruct Pulmon Dis. 2017 Aug 21;12:2465-75.

11. Yu JH, Shin M-S, Lee JR, Choi JH, Koh EH, Lee WJ, et al. Decreased sucrose preference in patients with type 2 diabetes mellitus. Diabetes Res Clin Pract. 2014 May;104(2):214-9.

12. Mojet J. Taste Perception with Age: Generic or Specific Losses in Threshold Sensitivity to the Five Basic Tastes? Chem Senses. 2001 Sep 1;26(7):845-60.

13. Donaldson LF, Bennett L, Baic S, Melichar JK. Taste and weight: is there a link? Am J Clin Nutr. 2009 Sep 1;90(3):800S-803S.

14. Umabiki M, Tsuzaki K, Kotani K, Nagai N, Sano Y, Matsuoka $Y$, et al. The Improvement of Sweet Taste Sensitivity with Decrease in Serum Leptin Levels During Weight Loss in Obese Females. Tohoku J Exp Med. 2010;220(4):267-71.

15. Jayasinghe S, Kruger R, Walsh D, Cao G, Rivers S, Richter M, et al. Is Sweet Taste Perception Associated with Sweet Food Liking and Intake? Nutrients. 2017 Jul 14;9(7):750. 